\title{
Properties of boron carbide nanotubes: Density-functional-based tight-binding calculations
}

\author{
O. Ponomarenko, M. W. Radny, ${ }^{*}$ and P. V. Smith \\ School of Mathematical and Physical Sciences, The University of Newcastle, Callaghan, NSW, 2308 Australia \\ G. Seifert \\ Institut für Physikalische Chemie, Technische Universität Dresden, D-01062 Dresden, Germany \\ (Received 16 September 2002; revised manuscript received 20 December 2002; published 4 March 2003)
}

\begin{abstract}
The existence of stable layered bulk allotropes can be an indication for the existence of corresponding stable tubular structures. Using a density functional tight binding method we demonstrate that charged borocarbide (BC) nanotubes, which are isoelectronic with layered lithium or copper borocarbide, are stable and energetically viable. The discussed structures are semiconducting with an energy gap which decreases with diameter. The size of the gap also depends on the helicity of the tubes, and is always smaller for tubes with armchair $(n, n)$ and zig-zag $(3 n, 0)$ symmetry than for tubes with other symmetries. Hole doping and its effects on the electronic structure of $\mathrm{BC}^{(-)}$layers and tubes is also discussed.
\end{abstract}

DOI: 10.1103/PhysRevB.67.125401

PACS number(s): 61.46.+w, 62.25.+g, 73.22.-f, 74.62.Dh

\section{INTRODUCTION}

Since the discovery of carbon nanotubes by Ijima ${ }^{1}$ in 1991 a broad range of tubular structures with different atomic composition have been investigated. Noncarbon nanotubes such as $\mathrm{BN},{ }^{2} \mathrm{BC}_{2} \mathrm{~N},{ }^{3} \mathrm{~B}_{x} \mathrm{C}_{y} \mathrm{~N}_{z},{ }^{4} \mathrm{BC}_{3},{ }^{3} \mathrm{CN}_{x},{ }^{5}$ and $\mathrm{GaSe}^{6}$ have been predicted to be stable, and some have been observed experimentally (for a recent review about nanotubes formed from inorganic materials see, for example, Tenne and $\left.Z^{2} t l^{7}\right)$. An analysis of synthesized noncarbon nanotubes such as $\mathrm{GaN}^{8}, \mathrm{WS}_{2}$ and $\mathrm{MoS}_{2},{ }^{9} \mathrm{NiCl}_{2},{ }^{10} \mathrm{SnS}_{2},{ }^{11} \mathrm{Te},{ }^{12}$ and $\mathrm{Bi}^{13}$ nanotubes has led to some generalizations regarding the necessary conditions for their stability and the pathways involved in their synthesis. ${ }^{14}$ The most important seems to be the ability of the material to form a layered threedimensional bulk structure.

Some of the high-temperature superconducting materials occur in a layered structure. ${ }^{15,16}$ Among them, metal borocarbides ${ }^{17}$ have attracted attention recently due to their potential to serve as a source for designing novel superconductors. ${ }^{18-23}$ Scandium boron carbide $\left(\mathrm{Sc}_{2} \mathrm{~B}_{1.1} \mathrm{C}_{3.2}\right)$, for instance, can be described as a $\mathrm{B}_{1 / 3} \mathrm{C}_{2 / 3}$ extended graphitelike layered material. ${ }^{16,24}$ Other examples of layered borocarbides include $\mathrm{CuBC}^{25}$ and $\mathrm{LiBC}^{21,26}$ which are isoelectronic with $\mathrm{MgB}_{2}{ }^{27}$ and have structural and bonding similarities with the "fully intercalated graphite structure" of this novel metallic layered, high temperature superconductor $\left(T_{C}\right.$ $\approx 40 \mathrm{~K})$. The electronic structure calculations of Rosner et al. ${ }^{26}$ suggest that hole doping of LiBC may result in strong electron-phonon coupling comparable to that in $\mathrm{MgB}_{2}$. As the electron-phonon coupling seems to be the main factor in the superconductivity of $\mathrm{MgB}_{2}$, hole doped $\mathrm{BC}$ systems may become prime candidates in the search for novel boron-based high-temperature superconductors. It has also been predicted that surface curvature may affect the electronic density of states (DOS) near the Fermi energy and increase the critical temperature $T_{C} \cdot{ }^{28}$ All of these observations have promoted our interest in investigating the possibility of layered $\mathrm{LiBC}$ forming tubular structures, and the extent to which the electronic properties of these structures might be modified by the constraints of the nanotube topology. Some attempts have been made to explore the plausibility of metal-boron nanotubes (see, for example, Ref. 29), inspired by crystalline diborides such as $\mathrm{AlB}_{2}$. In the present paper we discuss the properties of charged $\mathrm{BC}$ nanotubes $\left(\mathrm{BC}^{(-)}\right)$. The $\mathrm{BC}^{(-)}$ system, which is isoelectronic to $\mathrm{LiBC}$, can be useful in determining the properties of the boron-carbon hexagonal "back-bone" structure as a precursor for forming BC-metal doped nanotubes. The present study is the first step in this direction.

Nanotubes containing boron and carbon in different proportions have been extensively studied both theoretically and experimentally. It has been found experimentally ${ }^{30}$ that boron doping of carbon nanotubes leads to symmetry (helicity) selection during their synthesis. This is observed as an increased number of nanotubes with zigzag symmetry. Calculations by Hernandez et al. ${ }^{31}$ and Radny et al. ${ }^{32}$ have shown that the stability and symmetry selection of finite, borondoped single walled carbon nanotubes (SWCNT's) can be explained by the lowering of the "frustration energy" due to the chemisorption of boron atoms at the open ends of the tubes. The chemisorbed boron atoms change the balance between the strain and chemical (frustration) energies of the open ended finite tubes, and significantly lower the total energies of tubes of certain helicity. The properties of $\mathrm{BC}_{3},{ }^{4}$ $\mathrm{C}_{35} \mathrm{~B}^{33}$ and $\mathrm{B}_{x} \mathrm{C}(x \leqslant 0.10)^{34}$ single-walled nanotubes have also been experimentally observed and studied theoretically. In this paper we address the problem of the stability and electronic properties of charged $\mathrm{BC}^{(-)}$single layer nanotubes.

\section{ATOMIC STRUCTURE AND METHODOLOGY}

It is known that $\mathrm{LiBC}$ consists of hexagonal $\mathrm{BC}$ layers with alternating boron and carbon atoms and a B-C bond distance of $1.55 \AA .{ }^{35}$ The BC layers are separated by planes of $\mathrm{Li}$ atoms with a $\mathrm{Li}-\mathrm{BC}$ distance of $3.53 \AA$ (see Fig. 1). Since $\mathrm{Li}$ is a very electropositive element, it gives its electron to the more electronegative elements $(\mathrm{B}, \mathrm{C})$. This system is modeled in the present study by taking the BC layers 


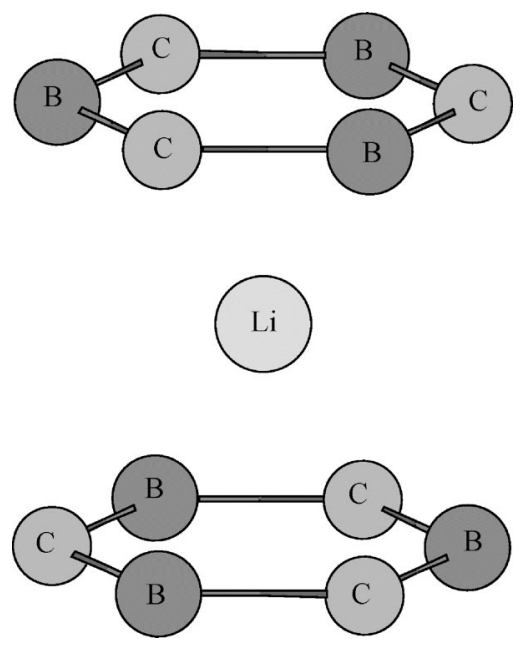

FIG. 1. LiBC layer structure.

to be charged $\left(\mathrm{BC}^{(-)}\right)$and treating the counter ions $\left(\mathrm{Li}^{+}\right)$as a homogeneously distributed background charge. While this modeling produces $\mathrm{BC}^{(-)}$structures which are isoelectronic to LiBC, it may exaggerate the ionicity of borocarbides. ${ }^{23}$ We have also considered holelike doping of $\mathrm{BC}^{(-)}$by having only one additional electron for every second boron or carbon atom. This reduces the charge of the borocarbide nanotube from $-e$ to $-0.5 e$ per boron (or carbon) atom and yields the configuration denoted here as $\mathrm{BC}^{(-0.5)}$.

The initial geometries of $\mathrm{BC}^{(-)}$and $\mathrm{BC}^{(-0.5)}$ tubular structures have been obtained by simply rolling up the corresponding sheets with alternating boron and carbon atoms placed on the nodes of the "honeycomb" lattice. The atomic interactions have been modeled using a nonorthogonal density-functional tight binding scheme (DFT-TB) due to Porezag et $a l .{ }^{36}$ This method employs an atomic basis consisting of four orbitals per atom with the Hamiltonian and overlap matrix elements calculated directly from density functional theory (DFT) within the local density approximation (LDA). This approach has been shown to be a good compromise between the more accurate, but more costly, $a b$ initio techniques, and cheaper to use, but less accurate, empirical potentials. Its applicability to nanotubes has already been shown for several tubular systems. ${ }^{37,38}$ For each $\mathrm{BC}^{(-)}$ tubular structure (with different helicity and radius) a series of calculations was carried out in which the initial structure was fully relaxed with respect to both the atomic positions and the tube cell length using the conjugated gradient technique. The stability of the formed tubes was also tested using canonical ensemble (NVT) molecular dynamics (MD) simulations. The electronic properties of the $\mathrm{BC}^{(-)}$planar layers and the resulting stable tubular structures were also calculated within the DFT-TB method.

\section{RESULTS AND DISCUSSION}

We have found that $\mathrm{BC}$ tubes are not stable without additional charge. Optimized $\mathrm{BC}^{(-)}$tubules based on a charged boron-carbon "skeleton," on the other hand, maintain a smooth cylindrical structure with a B-C bond length of

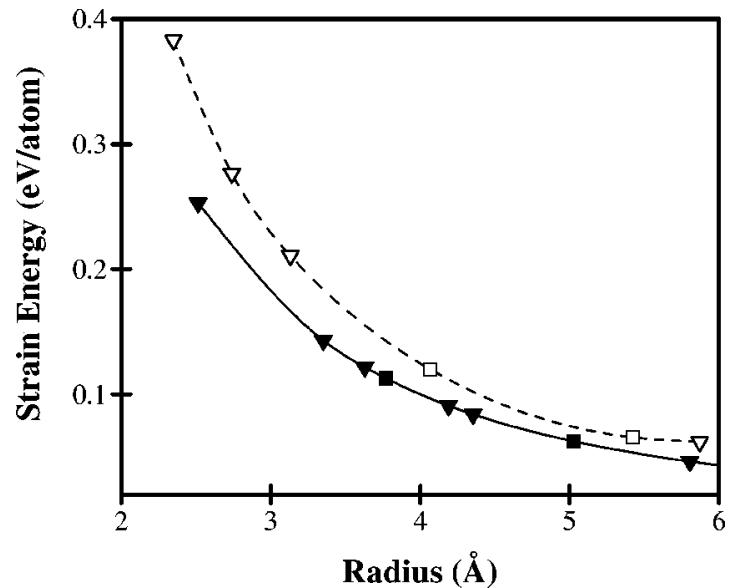

FIG. 2. Strain energies of single walled carbon nanotubes (empty symbols), and $\mathrm{BC}^{(-)}$nanotubes (filled symbols) as a function of the tube radius. The triangles correspond to $(n, 0)$ tubes and the squares to $(n, n)$ tubes.

$1.52-1.53 \AA$. These $\mathrm{BC}^{(-)}$tubular structures are also found to be preserved after extended MD simulations $(\sim 3-7 \mathrm{ps})$ at temperatures ranging from 500 to $1000 \mathrm{~K}$. The calculated strain energies of $\mathrm{BC}^{(-)}$tubes as a function of tube radius are shown in Fig. 2 and tabulated in Table I. The results demonstrate that the strain energy of a $\mathrm{BC}^{(-)}$tube, which is the difference between the energy of the tube and the energy of the corresponding layered structure (zero strain energy), converges approximately as $1 / D^{2}$ towards the value of the layered structure as the diameter $D$ increases. This behavior is similar to that of carbon nanotubes (CNT's), ${ }^{38}$ although the strain energies of the $\mathrm{BC}^{(-)}$tubes are clearly smaller than those of CNT's with comparable radii (see Fig. 2). The calculated strain energies of the $\mathrm{BC}^{(-)}$charged single walled nanotubes lie within the range of values for the energies of the experimentally observed nanotubes such as $\mathrm{BN}, \mathrm{C}_{x} \mathrm{~B}_{y} \mathrm{~N}_{z}$, and $\mathrm{BC}_{3}$. These results provide strong support for the possible existence of stable tubular $\mathrm{BC}^{(-)}$structures and

TABLE I. Calculated radii, strain energies, and electronic band gaps for $\mathrm{BC}^{(-)}$nanotubes.

\begin{tabular}{lccc}
\hline \hline $\begin{array}{l}\text { Geometry } \\
(m, n)\end{array}$ & $\begin{array}{c}\text { Radius } \\
(\AA)\end{array}$ & $\begin{array}{c}\text { Strain energy } \\
(\mathrm{eV} / \text { atom })\end{array}$ & $\begin{array}{c}\text { Band gap width } \\
(\mathrm{eV})\end{array}$ \\
\hline$(6,0)$ & 2.51 & 0.253 & 1.640 \\
$(7,0)$ & 2.93 & 0.187 & 2.125 \\
$(8,0)$ & 3.35 & 0.143 & 2.319 \\
$(5,5)$ & 3.63 & 0.122 & 2.212 \\
$(9,0)$ & 3.77 & 0.113 & 2.196 \\
$(10,0)$ & 4.19 & 0.091 & 2.238 \\
$(6,6)$ & 4.35 & 0.084 & 2.146 \\
$(12,0)$ & 5.03 & 0.062 & 2.106 \\
$(8,8)$ & 5.81 & 0.046 & 2.087 \\
$(14,0)$ & 5.87 & 0.045 & 2.160 \\
$(15,0)$ & 6.29 & 0.039 & 2.069 \\
$(12,12)$ & 8.71 & 0.019 & 2.040 \\
\hline \hline
\end{tabular}


(a)

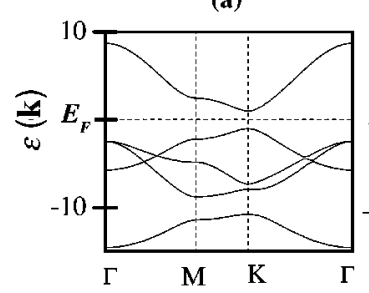

(c)

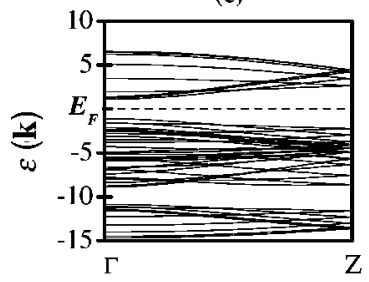

(e)

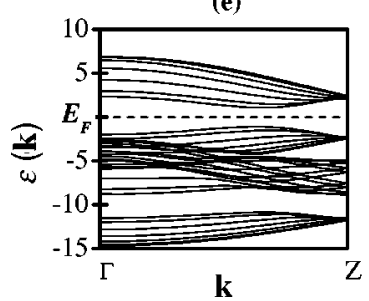

(b)

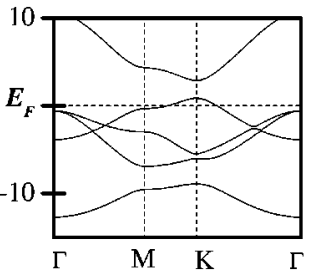

(d)

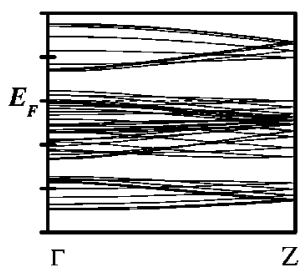

(f)

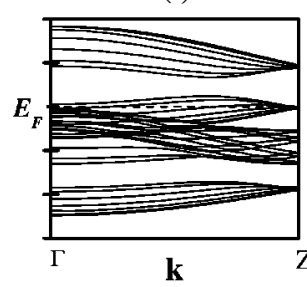

FIG. 3. The electronic band structures of (a) $\mathrm{BC}^{(-)}$and (b) $\mathrm{BC}^{(-0.5)}$ planar layers, (c) $(9,0) \mathrm{BC}^{(-)}$and $(\mathrm{d})(9,0) \mathrm{BC}^{(-0.5)}$ nanotubes, (e) $(6,6) \mathrm{BC}^{(-)}$and (f) $(6,6) \mathrm{BC}^{(-0.5)}$ nanotubes. All the energies are given relative to the Fermi energy $E_{F}$.

the formation of $\mathrm{BC}$-metal doped tubes analogous to $\mathrm{BN}$ and $\mathrm{BCN}$ tubes.

The electronic properties of $\mathrm{BC}^{(-)}$planar layers and the corresponding stable tubular structures have also been calculated within the DFT-TB method. The band structure for the layered $\mathrm{BC}^{(-)}$structure is shown in Fig. 3(a). As can be seen from the figure, the valence bands are separated from the conduction bands by $2.01 \mathrm{eV}$, with the valence band maximum and the conduction band minimum both occurring at the $K$ point. These basic features of the layered $\mathrm{BC}^{(-)}$band structure are very similar to the band structures of LiBC bulk materials. ${ }^{26}$ As examples of the electronic band structures of $\mathrm{BC}^{(-)}$tubes with different helicity, the electronic structures of zigzag $(9,0)$ and $(6,6)$ armchair tubes are shown in Figs. 3(c) and 3(e), respectively. The corresponding density of states (DOS) of $(9,0)$ and $(6,6)$ tubes are shown in Figs. 4(a), 4(c) and Figs. 4(b), 4(d), respectively. The DOS structure of the lower part of each valence band is characterized by a large contribution from C- $s$ and B- $s$ states, while the higher energy part of the valence band DOS is dominated by C- $p$ and $\mathrm{B}-p$ states. The conduction band is mainly characterized by C- $p$ and B- $p$ contributions. The structure of the calculated DOS is only weakly influenced by the chirality of the tubes as shown in Fig. 4.

We have found that the $\mathrm{BC}^{(-)}$tubes, similar to the layered $\mathrm{BC}^{(-)}$structures, are semiconducting. Figure 5 shows the size of the energy gap for $\mathrm{BC}^{(-)}$nanotubes with armchair and zigzag symmetry as a function of the tube radius. While the differences in the energy gaps are not large in absolute value, it is clear that this dependence is not smooth and is
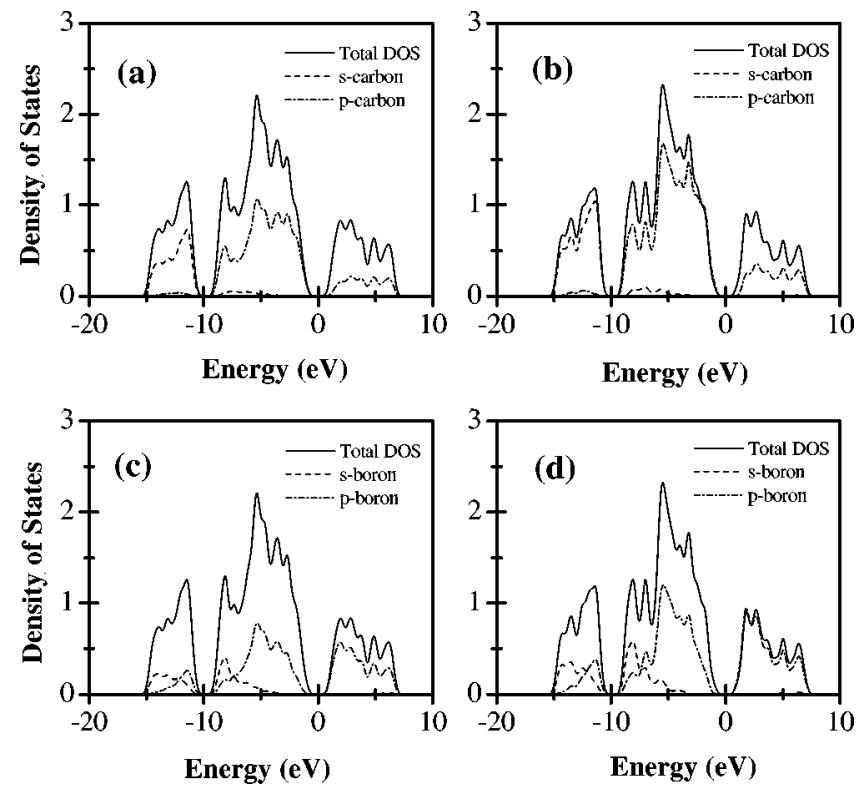

FIG. 4. The total and projected density of states (DOS) calculated for (a) and (c) $(9,0) \mathrm{BC}^{(-)}$nanotubes. (b) and (d) $(6,6) \mathrm{BC}^{(-)}$ nanotubes.

affected by both the helicity and the diameter of the tubes. The calculated values of the band gap for armchair $(n, n)$ and $(3 n, 0)$ zig-zag tubes are always smaller than tubes of comparable radius with helicities for which $m-n \neq 3 q$ ( $q$ is an integer). This is consistent with the folding model, ${ }^{39}$ which predicts that tubes satisfying the condition that $m-n$ is an integral multiple of 3 , should have the smallest energy gaps. The folding model also predicts that the size of the energy gap of such tubes should be equal to the energy gap at the $K$ point of the Brillouin zone of the corresponding boroncarbide layer. With the exception of the $(6,0)$ and $(7,0)$ nanotubes which have gaps of only 1.64 and $2.12 \mathrm{eV}$, respectively, the size of the gap is seen to decrease from about 2.32 $\mathrm{eV}$ for the $(8,0)$ tube towards the value of the flat $\mathrm{BC}^{(-)}$

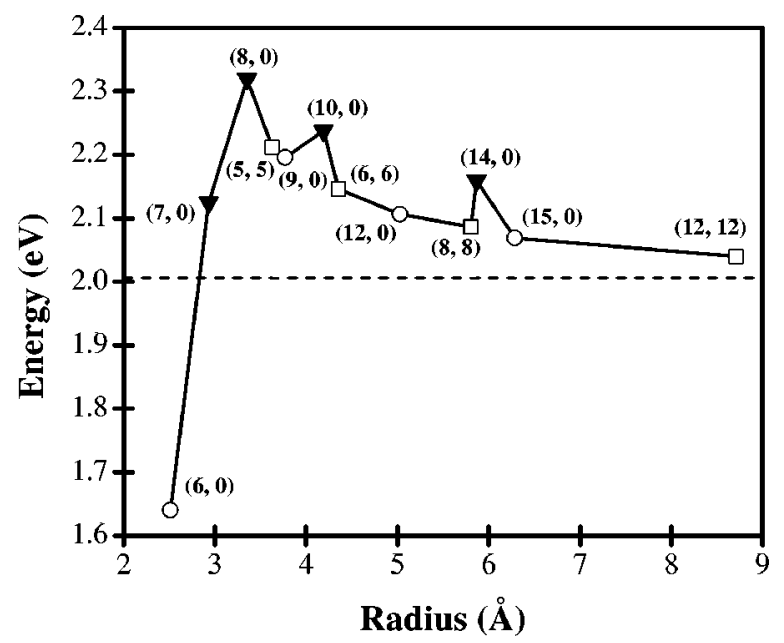

FIG. 5. Calculated gap-size versus radius for $\mathrm{BC}^{(-)}$nanotubes. The energy gap for the planar $\mathrm{BC}^{(-)}$structure is denoted by the single dashed line at $2.01 \mathrm{eV}$. 
layer $(2.01 \mathrm{eV})$ for large tubes such as $(12,12)$. This tendency of the size of the energy gap of $\mathrm{BC}^{(-)}$nanotubes to decrease with increasing radius is very similar to that of single walled CNT's $^{40}$ with helicities satisfying the relationship $m-n$ $\neq 3 q$ (the ideal armchair $(n, n)$ and $(3 n, 0)$ zig-zag CNT's are metallic with zero energy gap, in analogy with the corresponding graphene sheet). The deviations from this simple picture, which are shown by $\mathrm{BC}^{(-)}$nanotubes of small radius are presumably related to curvature (strain) effects. The calculated energy gaps of the $\mathrm{BC}^{(-)}$tubes as a function of the tube radius are given in Table $\mathrm{I}$.

It is interesting to compare the above determined behavior of $\mathrm{BC}^{(-)}$nanotubes with the electronic properties of other tubular structures derived from hexagonal layered materials. $\mathrm{BN}$ nanotubes are isoelectronic to both carbon and $\mathrm{BC}^{(-)}$ single walled nanotubes but, due to their higher ionicity, are wide-gap semiconductors. Rubio et al. ${ }^{2}$ have shown that the energy gaps of $\mathrm{BN}$ nanotubes increase with increasing diameter, and rapidly converge to the value of the hexagonal BN layer $(5.5 \mathrm{eV})$. As a result, $\mathrm{BN}$ tubes with radii larger than 6 $\AA$ are almost constant gap semiconductors with only a very small dependency on helicity. Single walled $\mathrm{BC}_{3}$ nanotubes, which are narrow gap semiconductors, also exhibit different energy gap behavior to $\mathrm{BC}^{(-)}$nanotubes. The energy gaps of $(n, 0) \mathrm{BC}_{3}$ nanotubes have been found to increase with increasing diameter and converge to the value of a flat $\mathrm{BC}_{3}$ sheet in the infinite limit, ${ }^{3}$ while $(n, n) \mathrm{BC}_{3}$ tubes have an almost constant energy gap, independent of their diameter. The different energy gap behavior of $\mathrm{BC}^{(-)}, \mathrm{BN}$ and $\mathrm{BC}_{3}$ single walled nanotubes may arise from a combination of the effects of ionicity, curvature, and buckling.

Rosner et $\mathrm{al}^{26}{ }^{2}$ suggested that hole doping of the LiBC structure, to produce sub-stoichiometric $\mathrm{Li}_{x} \mathrm{BC}$, should make this structure metallic. In order to study this effect in isolated layered and tubular $\mathrm{BC}^{(-)}$structures we have modeled the doping of the tubes with hole carriers by carrying out electronic structure calculations for $\mathrm{BC}^{(-0.5)}$ tubes with the same atomic structure as the $\mathrm{BC}^{(-)}$tubes. These $\mathrm{BC}^{(-0.5)}$ systems correspond to a concentration of cationic atoms such as $\mathrm{Li}$ in the $\mathrm{Li}_{x} \mathrm{BC}$ structure of $x=0.5$ (see Ref. 26). The calculated band structures of the layered and tubular $\mathrm{BC}^{(-0.5)}$ structures are shown in Figs. 3(b), 3(d), and 3(f). It can be seen that, while "hole doping" does not significantly change the topologies of the valence and conduction bands, the Fermi energy moves into the valence band, showing a rigid band behavior. As a result, the systems become metallic. This situation is again very similar to that of some of the substo- ichiometric $\mathrm{Li}_{x} \mathrm{BC}$ 3D structures discussed by Rosner et al. in Ref. 26.

These results may have some interesting consequences. Due to characteristic van Hove singularities in the DOS of tubular structures, shifting of the Fermi level into the valence band may result in a large value for the number of electronic states at the Fermi energy $N\left(E_{F}\right)$. This could have a direct effect on the electron-phonon coupling, similar to that in high-temperature superconductors. ${ }^{26}$ Indeed, the analysis of different structures including classical, mesoscopic, and novel high- $T_{C}$ superconductors has revealed the possibility of increasing the critical temperature in nanotubular structures by variation of the Fermi energy. Of course, this electronic DOS dominated picture of superconductivity, assuming other parameters and quantities remain constant, must be carefully investigated. For example, we have not considered the impact that cationic atoms (such as $\mathrm{Li}$ or $\mathrm{Cu}$ in the corresponding layered bulk material) may have on the strain energy, the interatomic stretching modes, and the underlying electronic structure. The energetics and electronic properties of layered borocarbide structures doped by lithium are currently being studied by our group.

\section{CONCLUSIONS}

Our calculations have shown that $\mathrm{BC}^{(-)}$tubes, as a prototype for BC-metal doped nanotubes, are energetically stable and could therefore prove to be viable structures. All of the calculated $\mathrm{BC}^{(-)}$tubes are semiconducting with the direct gap depending on the radius and helicity of the tube. The size of the energy gap for armchair $(n, n)$ and zig-zag $(3 n, 0) \mathrm{BC}^{(-)}$nanotubes is smaller than that for tubes with helicities satisfying the relationship $m-n \neq 3 q$. The calculated size of the energy gap decreases with radius and approaches the energy gap value of the corresponding $\mathrm{BC}^{(-)}$ layered structure. We have also modeled the doping of $\mathrm{BC}^{(-)}$ tubes. This has been found to make the tubes metallic by shifting the Fermi energy into the valence band.

\section{ACKNOWLEDGMENTS}

One of us, O.P., would like to thank the University of Newcastle (Australia) and the Technische Universität Dresden for financial support. We would also like to thank the AC3 supercomputing facility for processing time and the Space Physics Group of the University of Newcastle for access to some of their computer resources. G.S. also thanks the German Israeli Foundation (GIF) for support.
*Electronic address: Marian.Radny@ @ewcastle.edu.au

${ }^{1}$ S. Iijima, Nature (London) 354, 56 (1991).

${ }^{2}$ A. Rubio, J.L. Corkill, and M.L. Cohen, Phys. Rev. B 49, 5081 (1994).

${ }^{3}$ Y. Miyamoto, A. Rubio, S.G. Louie, and M.L. Cohen, Phys. Rev. B 50, 18360 (1994).

${ }^{4}$ Z. Weng-Sieh, K. Cherrey, N.G. Chopra, X. Blase, Y. Miyamoto, A. Rubio, M.L. Cohen, S.G. Louie, A. Zettl, and R. Gronsky, Phys. Rev. B 51, 11229 (1995).

${ }^{5}$ W.Q. Han et al., Appl. Phys. Lett. 77, 1807 (2000).
${ }^{6}$ M. Cote, M.L. Cohen, and D.J. Chadi, Phys. Rev. B 58, R4277 (1998).

${ }^{7}$ R. Tenne and A. K. Zettl, in Carbon Nanotubes, edited by M. S. Dresselhaus, G. Dresselhaus, and Ph. Avouris (Springer, Berlin, 2001), p. 81.

${ }^{8}$ S.M. Lee, Y.H. Lee, Y.G. Hwang, J. Elsner, D. Porezag, and T. Frauenheim, MRS Internet J. Nitride Semicond. Res. 4S1, G6.3 (1999).

${ }^{9}$ R. Tenne, L. Margulis, M. Genut, and G. Hodges, Nature (London) 360, 444 (1992). 
${ }^{10}$ Y.R. Hacohen, E. Grunbaum, R. Tenne, J. Sloan, and J.L. Hutchison, Nature (London) 395, 336 (1998).

${ }^{11}$ B. Alperson, M. Homyonfer, and R. Tenne, J. Electroanal. Chem. 473, 186 (1999).

${ }^{12}$ B. Mayers and Y.N. Xia, Adv. Mater. 14, 279 (2002).

${ }^{13}$ Y. Li, J. Wang, Z. Deng, Y. Wu, X. Sun, D. Yu, and P. Yang, J. Am. Chem. Soc. 123, 9904 (2001).

${ }^{14}$ R. Tenne, in Cluster Assembled Materials, edited by K. Sattler (Trans Tech Publications, Uetikon-Zurich, 1996), p. 275.

${ }^{15}$ J. van Duijn, J.P. Attfield, R. Watanuke, and K. Suzuki, J. Phys. Chem. Solids 62, 1423 (2001).

${ }^{16}$ Y. Shi, A. Leithe-Jasper, and T. Tanaka, Mol. Cryst. Liq. Cryst. 340, 271 (2000).

${ }^{17}$ J. Bauer, J.F. Halet, and J.Y. Saillard, Coord. Chem. Rev. 180, 723 (1998).

${ }^{18}$ R. Nagarajan, J. Low Temp. Phys. 107, 517 (1997).

${ }^{19}$ S.L. Drechsler et al., Physica C 341, 749 (2000).

${ }^{20}$ S.L. Drechsler et al., Physica C 364, 31 (2001).

${ }^{21}$ P. Ravindran, P. Vajeeston, R. Vidya, A. Kjekshus, and $\mathrm{H}$. Fjellvag, Phys. Rev. B 64, 224509 (2001).

${ }^{22}$ K.H. Muller and V.N. Narozhnyi, Rep. Prog. Phys. 64, 943 (2001).

${ }^{23}$ W.E. Pickett, Physica B 296, 112 (2001).

${ }^{24}$ T. Mori, Y. Shi, and T. Tanaka, Mol. Cryst. Liq. Cryst. 340, 83 (2000).

${ }^{25}$ M.J. Mehl, D.A. Papaconstantopoulos, and D.J. Singh, Phys. Rev. B 64, 140509 (2001).

${ }^{26}$ H. Rosner, A. Kitaigorodsky, and W.E. Pickett, Phys. Rev. Lett. 88, 127001 (2002).
${ }^{27}$ P. Zhang and V.H. Crespi, Phys. Rev. Lett. 89, 56403 (2002).

${ }^{28}$ E. Montevecchi and J.O. Indekeu, Europhys. Lett. 51, 661 (2000).

${ }^{29}$ A. Quandt, A.Y. Liu, and I. Boustani, Phys. Rev. B 64, 125422 (2001).

${ }^{30}$ X. Blase, J.-C. Charlier, A. De Vita, R. Car, Ph. Redlich, M. Terrones, W.K. Hsu, H. Terrones, D.L. Carroll, and P.M. Ajayan, Phys. Rev. Lett. 83, 5078 (1999).

${ }^{31}$ E. Hernandez, P. Ordejón, I. Boustani, A. Rubio, and J.A. Alonso, J. Chem. Phys. 113, 3814 (2000).

${ }^{32}$ M. W. Radny, V. Kumar, and Y. Kawazoe (unpublished).

${ }^{33}$ B.C. Satishkumar, A. Govindaraj, K.R. Harikumar, J.P. Zhang, A.K. Cheetham, and C.N.R. Rao, Chem. Phys. Lett. 300, 473 (1999).

${ }^{34}$ W.Q. Han, Y. Bando, K. Kurashima, and K. Sato, Chem. Phys. Lett. 299, 368 (1999).

${ }^{35}$ M. Wörle, R. Nesper, G. Mair, M. Schwarz, and H.G. von Schnering, Z. Anorg. Allg. Chem. 621, 1153 (1995).

${ }^{36}$ D. Porezag, Th. Frauenheim, Th. Köhler, G. Seifert, and R. Kaschner, Phys. Rev. B 51, 12947 (1995).

${ }^{37}$ G. Seifert, H. Terrones, M. Terrones, G. Jungnickel, and T. Frauenheim, Phys. Rev. Lett. 85, 146 (2000).

${ }^{38}$ E. Hernandez, C. Goze, P. Bernier, and A. Rubio, Phys. Rev. Lett. 80, 4502 (1998).

${ }^{39}$ R. Saito, G. Dresselhaus, and M. S. Dresselhaus, Physical Properties of Carbon Nanotubes (Imperial College Press, London, 1998), p. 59.

${ }^{40}$ C.T. White, D.H. Robertson, and J.W. Mintmire, Phys. Rev. B 47, 5485 (1993). 